\title{
A comparative study of the quality of non- stimulated and stimulated tears in normal eye male subjects using the tear ferning test
}

This article was published in the following Dove Press journal: Clinical Optometry

\author{
Saud A Alanazi \\ Mohammed A Aldawood \\ Yousef S Badawood \\ Gamal A El-Hiti \\ Ali M Masmali \\ Cornea Research Chair, Department of \\ Optometry, College of Applied Medical \\ Sciences, King Saud University, Riyadh \\ 1 1433, Saudi Arabia
}

Purpose: To evaluate and compare the tear ferning patterns of non-stimulated and stimulated tears in normal eye male subjects using the tear ferning test.

Methods: Thirty-five male subjects aged 20-38 years with normal eyes were enrolled in the study. Subjects with dry eye or with a risk factor for eye dryness (eg, recent ocular surgery, contact-lens wearers, smokers, anemic, diabetes, and thyroid gland problems) were excluded from the study. Dry eye subjects were excluded based on the ocular surface diseases index scores, non-invasive tear break-up time, and tear meniscus height measurements from the right and left eyes. A tear sample was collected from both eyes in each subject to perform the tear ferning test for non-stimulated tears. After 10 mins, the tears were stimulated using a Schirmer strip, a tear sample was collected from both eyes in each participant, the tear ferning test was performed, and the ferns were graded based on the five-point tear ferning grading scale.

Results: The average tear ferning grade [median (interquartile range)] for non-stimulated tears from the right and left eyes was 0.60 (1.25) and 0.50 (1.31), respectively. For stimulated tears, the average tear ferning grade from the right and left eyes was 0.50 (1.05) and 0.75 (1.25), respectively. There was no significant difference in the tear ferning grades obtained from non-stimulated and stimulated tears (Wilcoxon test, $P>0.05$ ).

Conclusion: This study found no significant difference in the tear ferning patterns obtained from non-stimulated and stimulated tears, or between the tear ferning patterns in the tears collected from the right and left eyes before and after stimulating tears. It seems that there were no changes in the chemical composition of tears before and after stimulation, possibly due to the steady concentration of electrolytes and lipids within the tear film for both nonstimulated and stimulated tears.

Keywords: tear ferning patterns, tear quality, tear film, eye dryness, non-stimulated and stimulated tears

\section{Introduction}

Tear film consists of three layers known as the mucin (inner), aqueous (middle), and lipid (outer) layers and has a complex structure. ${ }^{1-3}$ The mucin layer is produced by goblet cells and the lacrimal glands, while the meibomian glands are responsible for the production of lipids. ${ }^{4,5}$ Tear film covers the anterior surface of the eye, protects the cornea, provides the cornea with oxygen, lubricates and moistens the eye surface, and washes foreign bodies from the eye. ${ }^{5}$ The lipid layer influences tear film stability, and dysfunction of meibomian gland secretions leads to changes in
Gamal A El-Hiti

Department of Optometry, College of Applied Medical Sciences, King Saud

University, P.O. Box 10219 , Riyadh II433, Saudi Arabia

Tel +966 II $4693543 ;+966$ II 4693778

Email saaalanazi@ksu.edu.sa;

gelhiti@ksu.edu.sa 
both the quality and quantity of tears. ${ }^{6}$ Instability in the tear film leads to a dry eye, a disturbance in the visual process, causing eye pain, dryness, and inflammation, and negatively affecting the quality of life in $10-30 \%$ of the global population. ${ }^{7}$ The cost of the treatment of dry eye syndrome across the globe is estimated to be over $\$ 100$ billion. ${ }^{8,9}$ Thus, many studies have been conducted on the tear film, and on the lipid layer in particular, to investigate which factors could negatively affect the ocular surface. ${ }^{10}$ Such studies found strong associations between the normal structure of tear film and thickness of the lipids layer, smoothness of the ocular surface, low evaporation rate of tears, high viscosity of tears, and the efficiency of ocular surface as antimicrobial barrier. ${ }^{5}$

Deficiency in tear secretion is common in approximately $50 \%$ of patients who experience dry eye symptoms, which is mainly due to dysfunction of the lacrimal glands. ${ }^{6}$ The aqueous layer of the tear film is water-rich and contains various types of proteins and electrolytes. The regulation of these tear proteins and electrolytes is vital to the control of tear secretion. ${ }^{11,12}$ However, a high tear evaporation rate is common due to dysfunction of the meibomian glands, leading to a reduction in lipid production. ${ }^{13}$ The quality and quantity of tears can be measured using various tests. The most common diagnostic tests used to assess the quantity of tears are the phenol red thread, ${ }^{14}$ Schirmer, ${ }^{14}$ tear meniscus height $(\mathrm{TMH}){ }^{15}$ and tear evaporation rate ${ }^{16}$ tests. Other tests can be used to detect the quality of tears, such as tear ferning (TF), ${ }^{17,18}$ osmolarity, ${ }^{19}$ and non-invasive tear break-up time (NITBUT). ${ }^{20}$ In addition, dry eye symptoms can be assessed with the use of dry eye questionnaires such as the ocular surface disease index (OSDI). ${ }^{21}$ Eye drops can be used to improve the tear film stability in patients with moderate eye dryness. ${ }^{22}$ Further, mechanical massage and warming eye lid devices could help patients with reduced lipid secretion. ${ }^{23,24}$

The TF pattern reflects the chemical composition of tears when dried. The five-points grading scale includes five TF grades (grades 0 to 4 ) in which grade 0 shows a TF pattern with no gaps or spaces between the branches and ferns. ${ }^{18}$ Grade 1 TF pattern shows the presence of small gaps between the branches ferns. The gaps and the thickness of the branches increased in Grade 2 and increased further in Grade 3. ${ }^{18}$ Grade 4 shows no TF pattern at all. ${ }^{18}$ Recently, we demonstrated that the TF test can be used to detect eye dryness along with other diagnostic tests. ${ }^{25-29}$ The TF test was proved to be a valid and a reliable to detect eye dryness. ${ }^{30}$ To our knowledge, the TF test has not been used to compare the quality of non-stimulated and stimulated tears among normal eye subjects. In addition, the TF patterns provide information about the chemical composition in non-stimulated and stimulated tears. Therefore, the purpose of the current research was to evaluate and compare the TF patterns of non-stimulated and stimulated tears obtained from both the left and right eyes of normal eye subjects.

\section{Materials and methods Subjects}

Thirty-five male subjects aged 20-38 years (mean \pm standard deviation $=23.36 \pm 2.48$ years) with normal eyes were enrolled in this study. The subjects were students at the College of Applied Medical Sciences, King Saud University, Riyadh. Subjects with dry eyes or a risk factor for eye dryness (eg, those who had undergone recent ocular surgery or who were contact-lens wearers, smokers, anemic, had diabetes, had high body mass index, high cholesterol, or had thyroid gland problems) were excluded from the study. Dry eye subjects were excluded based on the OSDI score, NITBUT, and TMH measurements from the right and left eyes. Ethical approval was obtained from the College of Applied Medical Sciences Ethics Committee, King Saud University. Written informed consent was obtained from each participant prior to the commencement of the research and the subjects were treated according to the tenets of Helsinki Declaration. ${ }^{31}$ All tests were performed by the same examiner in a controlled environment at a temperature of $23^{\circ} \mathrm{C}$ and a humidity of $<40 \%$. The OSDI was completed by each participant followed by the TF, TMH, and NITBUT tests. A 10 mins rest period was allowed between tests.

\section{OSDI}

The OSDI was first completed by each participant and a score of $<13$ was considered indicative of a normal eye. ${ }^{21}$

\section{TMH and NITBUT tests}

The TMH and NITBUT tests were performed on OCULUS Keratograph ${ }^{\circledR} 4$ (OCULUS Inc., Wetziar, Germany). Fluorescein was added to the subject's eye. For NITBUT, the subject was asked to refrain from blinking while the tear film was observed. A yellow barrier filter was used to enhance the visibility of the tear film breakup. The tear break-up time was recorded as the number of seconds that elapsed between the last blink and the 
appearance of the first dry spot in the tear film. The inferior TMH images were captured and measured perpendicular to the lid margin at the central point relative to the pupil center using an integrated ruler $(\mathrm{mm})$. A lower lid TMH of $>0.2 \mathrm{~mm}$ and tear break-up time of $>10 \mathrm{~s}$ were indicative of a normal eye.

\section{TF test}

A glass capillary tube $(10 \mu \mathrm{L})$ purchased from Merck (Schnelldorf, Germany) was used to collect a tear sample (1 $\mu \mathrm{L}$ ) from the lower meniscus of both eyes in each subject. The tear sample was dried at $23^{\circ} \mathrm{C}$ (humidity $<40 \%$ ) for 10 mins. An Olympus DP72 digital microscope (Tokyo, Japan) at a magnification power of $10 \times$ was used to observe the TF patterns. The test was repeated 10 mins later after stimulating the tears using a Schirmer strip. The TF patterns were graded based on the five-point TF grading scale in 0.1 increments. ${ }^{18}$ Normal eye was defined for a TF grade less than $2 .{ }^{18}$ The tears were collected by the same investigator and the TF patterns were grades by three investigators and the averages were calculated.

\section{Statistical analysis}

The data were analyzed using SPSS software (version 22; IBM, Armonk, NY, USA). A Spearman correlation coefficient $(r)$ was used to investigate the relationship between the obtained data. The correlation is considered as weak for $r=0.10-0.29$, medium for $r=0.30-0.49$, and strong for $r=0.50-1.00 .^{32}$ The data obtained from the OSDI and TMH, NITBUT, and TF tests were found to be non-normally distributed; therefore, the average scores are expressed as the median (interquartile range; IQR).

\section{Results}

The median scores for the OSDI and the TMH and NITBUT measurements obtained from both the right and left eyes are recorded in Table 1. There were no significant differences between the scores collected from the OSDI and the measurements obtained from TMH and NITBUT for both the right and left eyes (Wilcoxon test, $P>0.05$ ). The median (IQR) TF grades for the non-stimulated and stimulated tears obtained from the right and left eyes in each subject are reported in Table 2. There were no significant differences between the TF grades obtained from non-stimulated and stimulated tears and those obtained from both the right and left eyes (Wilcoxon test, $P>0.05)$. Box plots for the TF grades for the non-stimulated and stimulated tears in the right and left eyes for the
Table I Ocular surface disease index, tear meniscus height, noninvasive tear break-up time, and tear ferning scores

\begin{tabular}{|l|l|}
\hline Test & Median (IQR) \\
\hline OSDI & $40.00(6.00)$ \\
TMH (OD; mm)* & $0.25(0.06)$ \\
TMH (OS; mm)* & $0.25(0.05)$ \\
NITBUT (OD; s)* & $9.80(5.33)$ \\
NITBUT (OS; s)* & $10.00(5.90)$ \\
\hline
\end{tabular}

Note: $* P>0.05$.

Abbreviations: IQR, interquartile range; OSDI, ocular surface disease index; TMH, tear meniscus height; NITBUT, non-invasive tear break-up time; OD, right eye; OS, left eye.

Table 2 Tear ferning grades

\begin{tabular}{|l|l|l|}
\hline \multirow{2}{*}{ TF grade* } & \multicolumn{2}{|l|}{ Median (IQR) } \\
\cline { 2 - 3 } & Non-stimulated tears & Stimulated tears \\
\hline TF (OD) & $0.55(0.89)$ & $0.65(0.99)$ \\
TF (OS) & $0.50(1.1 \mathrm{I})$ & $0.70(1.15)$ \\
\hline
\end{tabular}

Notes: $* P>0.05$. TF patterns were graded using 0.1 increments.

Abbreviations: IQR, interquartile range; TF, tear ferning; OD, right eye; OS, left eye.

study group $(n=35)$ are provided in Figure 1 . Representative TF images obtained from the right and left eyes for non-stimulated and stimulated tears obtained from the same subject are provided in Figures 2 and 3, respectively.

The correlations among the scores collected from the OSDI and TMH and NITBUT measurements in the right and left eyes are recorded in Table 3. There were strong correlations between the TMH $(r=0.577, P=0.001)$ and NITBUT $(r=0.610, P=0.001)$ measurements in the right and left eyes. No correlations were found among the OSDI scores and the TMH and NITBUT measurements.

The correlations between the TF grades for non-stimulated and stimulated tears obtained from the right and left eyes are recorded in Table 4 . There was a strong ( $r=0.554$, $P=0.007)$ correlation between the TF grades for non-stimulated tears obtained from the right and left eyes. In addition, there was a medium $(r=0.491, P=0.002)$ correlation between the TF grades for stimulated tears obtained from the right and left eyes.

\section{Discussion}

The current study suggested that there were no significant differences between the TF grades obtained from nonstimulated and stimulated tears collected from both the right and left eye of the study subjects $(P>0.05)$. A previous study conducted on non-stimulated $(n=17)$ and 


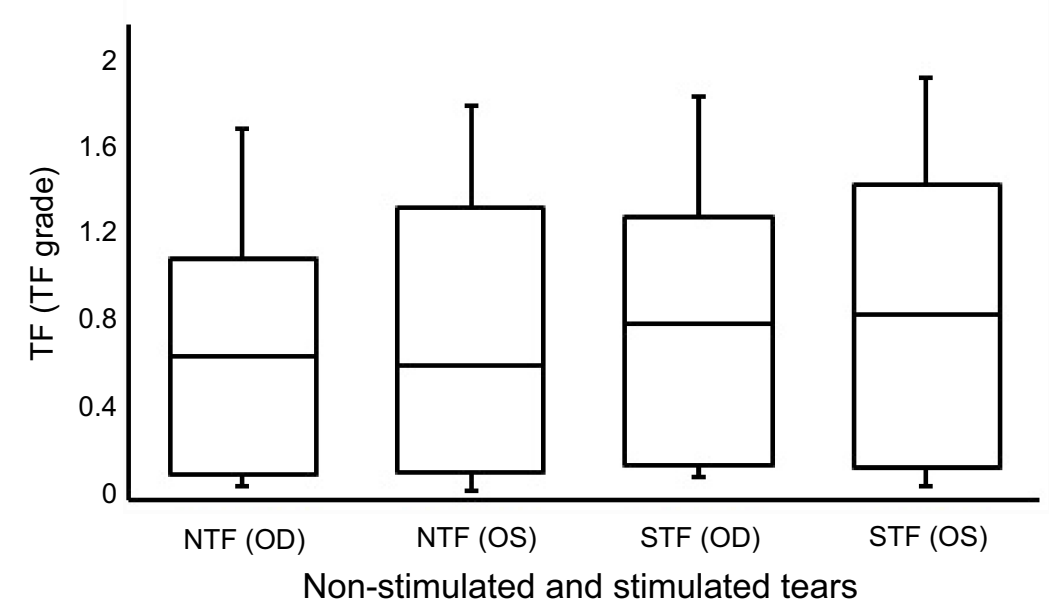

Figure I Box plots for the tear ferning grades for non-stimulated and stimulated tears.

Note: TF patterns were graded using 0.1 increments.

Abbreviations: TF, tear ferning; NTF (OD), tear ferning for non-stimulated tears collected from the right eye; NTF (OS), tear ferning for non-stimulated tears collected from the left eye; STF (OD), tear ferning for stimulated tears collected from the right eye; STF (OS), tear ferning for stimulated tears collected from the left eye.
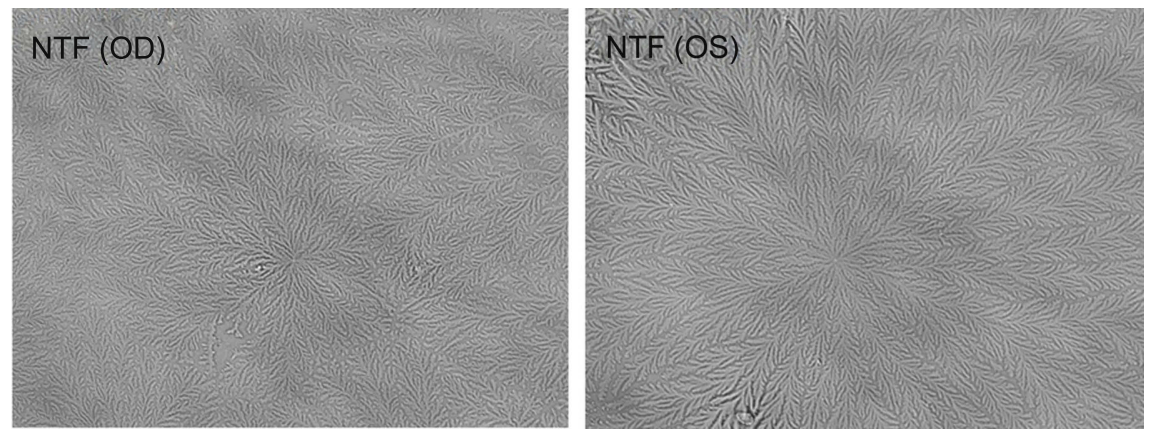

Figure 2 Representative tear ferning images of non-stimulated tears collected from the right and left eyes of the same subject.

Abbreviations: NTF (OD), tear ferning for non-stimulated tears collected from the right eye; NTF (OS), tear ferning for non-stimulated tears collected from the left eye.
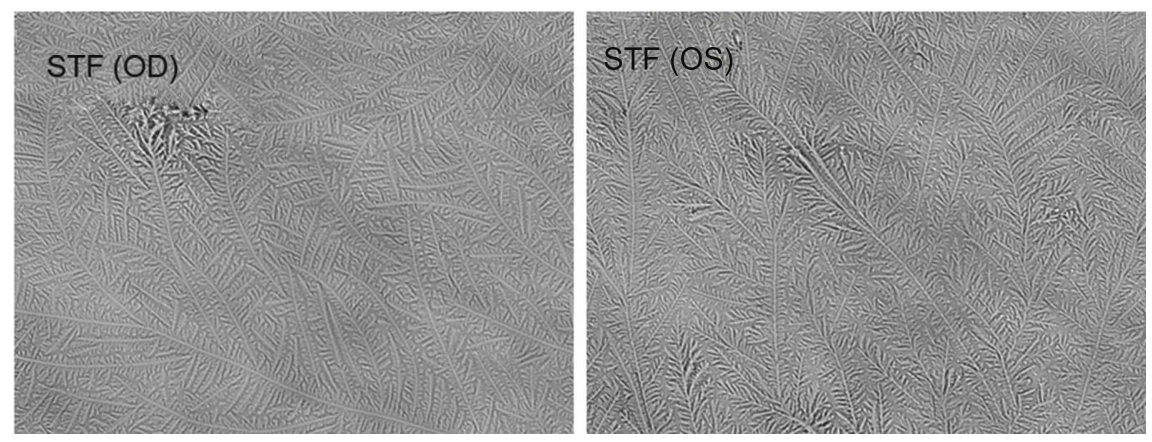

Figure 3 Representative tear ferning images of stimulated tears collected from the right and left eyes of the same subject.

Abbreviations: STF (OD), tear ferning for stimulated tears collected from the right eye; STF (OS), tear ferning for stimulated tears collected from the left eye.

stimulated $(n=19)$ tears found no significant difference in the viscosity of tears. ${ }^{33}$ In addition, the surface tension for non-stimulated $(43.0 \pm 2.1)$ and stimulated $(46.0 \pm 1.5)$ tears were similar. ${ }^{33}$ Such results suggested that the viscosity and surface tension are not dependent on the mucin level. ${ }^{33}$ Therefore, the mucin level does not play a primary role in the differentiation of non-stimulated and stimulated tears. ${ }^{33}$ Another study suggested that both non-stimulated and stimulated tears have the same concentration of mucins, and therefore have similar viscosity. ${ }^{34}$ 
Table 3 Correlations among the ocular surface disease index, tear meniscus height, and non-invasive tear break-up time scores

\begin{tabular}{|l|l|l|l|l|l|}
\hline Test/Correlation & OSDI & TMH (OD) & TMH (OS) & NITBUT (OD) & NITBUT (OS) \\
\hline OSDI & & & & & \\
$r$ & 1 & -0.002 & 0.055 & -0.239 & \\
$P$ & - & 0.992 & 0.743 & 0.149 & 0.004 \\
\hline TMH (OD) & & & & & 0.981 \\
$r$ & -0.002 & 1 & $0.577^{*}$ & 0.092 & \\
$P$ & 0.992 & - & 0.001 & 0.581 & 0.151 \\
\hline TMH (OS) & & & & & 0.364 \\
$r$ & 0.055 & $0.577^{*}$ & 1 & 0.057 & 0.183 \\
$P$ & 0.732 & 0.001 & - & 0.732 & 0.272 \\
\hline NITBUT (OD) & & & & & \\
$r$ & -0.239 & 0.092 & 0.057 & 1 & $0.610^{*}$ \\
$P$ & 0.144 & 0.581 & 0.732 & - & 0.001 \\
\hline NITBUT (OS) & & & & & 1 \\
$r$ & 0.004 & 0.151 & 0.183 & $0.610^{*}$ & - \\
$P$ & 0.981 & 0.364 & 0.272 & 0.001 & \\
\hline
\end{tabular}

Note: *Correlation is significant at the 0.01 level (two-tailed).

Abbreviations: $r$, Spearman correlation coefficient; $P$, significance; OSDI, ocular surface disease index; TMH, tear meniscus height; NITBUT, non-invasive tear break-up time; OD, right eye; OS, left eye.

Non-stimulated and stimulated tears collected from 30 normal eye subjects were previously analyzed by highperformance liquid chromatography, in which 12 types of

Table 4 Correlations among the TF grades obtained from nonstimulated and stimulated tears

\begin{tabular}{|l|l|l|l|l|}
\hline \multirow{2}{*}{ Test/Correlation } & \multicolumn{2}{|l|}{$\begin{array}{l}\text { Non-stimulated } \\
\text { tears }\end{array}$} & \multicolumn{2}{l|}{ Stimulated tears } \\
\cline { 2 - 5 } & $\begin{array}{l}\text { TF } \\
\text { (OD) }\end{array}$ & $\begin{array}{l}\text { TF } \\
\text { (OS) }\end{array}$ & $\begin{array}{l}\text { TF } \\
\text { (OD) }\end{array}$ & $\begin{array}{l}\text { TF } \\
\text { (OS) }\end{array}$ \\
\hline $\begin{array}{l}\text { Non-stimulated tears } \\
\text { TF (OD) }\end{array}$ & & & & \\
$r$ & 1 & $0.554^{*}$ & 0.275 & 0.291 \\
$P$ & - & 0.007 & 0.094 & 0.076 \\
\hline TF (OS) & & & & \\
$r$ & $0.554^{*}$ & 1 & 0.262 & 0.275 \\
$P$ & 0.007 & - & 0.112 & 0.095 \\
\hline Stimulated tears & & & & \\
TF (OD) & & & & \\
$r$ & 0.275 & 0.262 & $I$ & $0.491^{*}$ \\
$P$ & 0.094 & 0.112 & - & 0.002 \\
\hline TF (OS) & & & & \\
$r$ & 0.291 & 0.275 & $0.49 I^{*}$ & 1 \\
$P$ & 0.076 & 0.095 & 0.002 & - \\
\hline
\end{tabular}

Note: *Correlation is significant at the 0.01 level (2-tailed). TF patterns were graded using 0.1 increments.

Abbreviations: $r$, Spearman correlation coefficient; $P$, significance; TF, tear ferning; $\mathrm{OD}$, right eye; OS, left eye. protein were identified. ${ }^{35}$ The concentrations of lacrimal gland proteins, tear-specific prealbumin, lysozyme, and lactoferrin were similar in non-stimulated and stimulated tears. ${ }^{35}$ However, the remaining types of protein, such as immunoglobulin $\mathrm{A}$ and immunoglobulin $\mathrm{M}$, were higher in non-stimulated tears than in stimulated tears. ${ }^{34}$ Such results suggested that the rate of tear flow affects the produced protein profile. ${ }^{35}$ On the other hand, an enzyme-linked immunosorbent assay indicated that the concentrations of lysozyme, lactoferrin, serum albumin, and immunoglobulin A were similar in non-stimulated tears $(n=12) .{ }^{36}$ Clearly, the control of the rate of tear flow in the collection process is important.

High-flow mechanical and intense cooling pulse stimulation of the cornea leads to a significant increase in tear secretion $(P<0.05)$, while stimulation of the conjunctiva is not effective. ${ }^{37}$ However, moderate mechanical and cold stimulation of either the conjunctiva or the cornea does not significantly alter tear secretion. ${ }^{37}$ Electrical stimulation of the cornea, ${ }^{38}$ anterior ethmoid nerve, ${ }^{39,40}$ lacrimal gland, ${ }^{40}$ and lacrimal nerve ${ }^{41}$ leads to increased tear volume, and normal tear proteins and lipids, and a reduction in the osmolarity of tears. ${ }^{42}$ For example, electrical $(30-90 \mathrm{~Hz})$ stimulation of the anterior ethmoid nerve leads to a 92 $133 \%$ increase in tear volume $(P \leq 0.01) .{ }^{42}$ The level of tear secretion returned to the baseline volume after 7 mins of stimulation. The osmolarity of the tear film was reduced 
by $7 \mathrm{mOsmol} / \mathrm{L}$ and the concentration of both tear lipids (24-36\%) and proteins $(48 \%, P \leq 0.05)$ was increased. ${ }^{42}$ In addition, regular ( $\geq 4$ times daily) electrical stimulation of the anterior nerve can reduce dry eye symptoms. ${ }^{43}$ Thus, tear stimulation can improve tear secretion, but does not affect the quality or chemical composition of the tears. In addition, the stimulation does not alter the component of tears, as the current study suggested. Indeed, the average scores for the Schirmer test were found to be significantly higher in tears stimulated with a nasal electrical device than in non-stimulated tears in dry eye subjects $(n=40){ }^{39}$

\section{Limitations}

The current study has some limitations such as the relatively small number of subjects, and the participants were all normal eye males who reside in Riyadh City. Therefore, future studies should involve a larger sample size that contains both males and females and normal and dry eye subjects, and represent different geographic areas within Saudi Arabia.

\section{Conclusions}

The current study found no significant difference in the tear ferning patterns obtained from non-stimulated and stimulated tears of male subjects in Riyadh City. In addition, there were no significant differences between the tear ferning patterns of the tears collected from the right and left eyes before and after stimulating tears. It seems that there were no changes in the chemical composition of tears before and after stimulation, possibly due to the steady concentration of electrolytes and lipids within the tear film for both non-stimulated and stimulated tears. The tear ferning test has the potential to be used in clinics to assess the quality of tears.

\section{Abbreviation list}

$\mathrm{TMH}$, tear meniscus height; TF, tear ferning; NITBUT, non-invasive tear break-up time; OSDI, ocular surface disease index; IQR, interquartile range; $r$, Spearman correlation coefficient; OD, right eye; OS, left eye.

\section{Ethics approval and informed consent}

Written informed consent was obtained from each participant prior to the commencement of the research, and the subjects were treated according to the tenets of Helsinki Declaration.

\section{Acknowledgments}

This work was financially supported by the Deanship of Scientific Research, King Saud University, through the Vice Deanship of Scientific Research Chairs.

\section{Disclosure}

The authors report no conflicts of interest in this work.

\section{References}

1. Conrady CD, Joos ZP, Patel BC. Review: the lacrimal gland and its role in dry eye. J Ophthalmol. 2016;2016:7542929. doi:10.1155/ 2016/7542929

2. Govindarajan B, Gipson IK. Membrane-tethered mucins have multiple functions on the ocular surface. Exp Eye Res. 2010;90(6):655663. doi:10.1016/j.exer.2010.02.014

3. Montés-Micó R, Cerviño A, Ferrer-Blasco T, García-Lázaro S, Madrid-Costa D. The tear film and the optical quality of the eye. Ocul Surf. 2010;8(4):185-192. doi:10.1016/S1542-0124(12)70 233-1

4. Tsai PS, Evans JE, Green KM, et al. Proteomic analysis of human meibomian gland secretions. Br J Ophthalmol. 2006;90(3):372-377. doi:10.1136/bjo.2005.080846

5. Georgiev GA, Eftimov P, Yokoi N. Structure-function relationship of tear film lipid layer: a contemporary perspective. Exp Eye Res. 2017;163:17-28. doi:10.1016/j.exer.2017.03.013

6. Lemp MA, Crews LA, Bron AJ, Foulks GN, Sullivan BD. Distribution of aqueous-deficient and evaporative dry eye in a clinic-based patient cohort: a retrospective study. Cornea. 2012;31 (5):472-478. doi:10.1097/ICO.0b013e318225415a

7. Gayton JL. Etiology, prevalence, and treatment of dry eye disease. Clin Ophthalmol. 2009;3:405-412. doi:10.2147/OPTH.S5555

8. Yu J, Asche CV, Fairchild CJ. The economic burden of dry eye disease in the United States: a decision tree analysis. Cornea. 2011;30(4):379-387. doi:10.1097/ICO.0b013e3181f7f363

9. McDonald M, Patel DA, Keith MS, Snedecor SJ. Economic and humanistic burden of dry eye disease in Europe, North America, and Asia: a systematic literature review. Ocul Surf. 2016;14(2):144167. doi:10.1016/j.jtos.2015.11.002

10. Butovich IA. Lipidomics of human meibomian gland secretions: chemistry, biophysics, and physiological role of meibomian lipids. Prog Lipid Res. 2011;50(3):278-301. doi:10.1016/j.plipres.2011. 03.003

11. Dartt DA. Neural regulation of lacrimal gland secretory processes: relevance in dry eye diseases. Prog Retin Eye Res. 2009;28(3):155177. doi:10.1016/j.preteyeres.2009.04.003

12. Ding C, Walcott B, Keyser KT. Sympathetic neural control of the mouse lacrimal gland. Invest Ophthalmol Vis Sci. 2003;44(4):15131520. doi:10.1167/iovs.02-0406

13. Knop E, Knop N, Millar T, Obata H, Sullivan DA. The international workshop on meibomian gland dysfunction: report of the subcommittee on anatomy, physiology, and pathophysiology of the meibomian gland. Invest Ophthalmol Vis Sci. 2011;52(4):1938-1978. doi:10.1167/iovs.10-6997c

14. Masmali A, Alqahtani TA, Alharbi A, El-Hiti GA. Comparative study of repeatability of phenol red thread test versus Schirmer's test in normal adults in Saudi Arabia. Eye Contact Lens. 2014;40(3):127131. doi:10.1097/ICL.0000000000000025

15. Ibrahim OM, Dogru M, Takano Y, et al. Application of Visante optical coherence tomography tear meniscus height measurement in the diagnosis of dry eye disease. Ophthalmology. 2010;117 (10):1923-1929. doi:10.1016/j.ophtha.2010.01.057 
16. Abusharaha A, Alturki AA, Alanazi SA, et al. An assessment of the tear evaporation rate in thyroid gland patients. Clin Ophthalmol. 2019;13:131-135. doi:10.2147/OPTH.S188614

17. Masmali AM, Al-Qhtani S, Al-Gasham TM, El-Hiti GA, Purslow C, Murphy PJ. Application of a new grading scale for tear ferning in non-dry eye and dry eye subjects. Cont Lens Anterior Eye. 2015;38 (1):39-43. doi:10.1016/j.clae.2014.09.007

18. Masmali AM, Murphy PJ, Purslow C. Development of a new grading scale for tear ferning. Cont Lens Anterior Eye. 2014;37(3):178-184. doi:10.1016/j.clae.2013.09.011

19. Masmali A, Alrabiah S, Alharbi A, El-Hiti GA, Almubrad T. Investigation of tear osmolarity using the TearLab ${ }^{\mathrm{TM}}$ osmolarity system in normal adults in Saudi Arabia. Eye Contact Lens. 2014;40(2):74-78. doi:10.1097/ICL.0000000000000002

20. Mzumara T, Afonne J. Assessing the relationship between non-invasive tear break-up time and maximum blink interval values among young adults at Mzuzu University. Clin Optom. 2018;10:87-91. doi:10.2147/OPTO.S164466

21. Schiffman RM, Christianson MD, Jacobsen G, Hirsch JD, Reis BL. Reliability and validity of the ocular surface disease index. Arch Ophthalmol. 2000;118(5):615-621. doi:10.1001/archopht.118.5.615

22. Cuevas P, Outeiriño LA, Azanza C, Angulo J, Giménez-Gallego G. Improvement in the signs and symptoms of dry eye disease with dobesilate eye drops. Mil Med Res. 2015;2:35. doi:10.1186/s40779-015-0068-8

23. Goto E, Monden Y, Takano Y, et al. Treatment of non-inflamed obstructive meibomian gland dysfunction by an infrared warm compression device. $\mathrm{Br} J$ Ophthalmol. 2002;86(12):1403-1407. doi:10.1136/bjo.86.12.1403

24. Olson MC, Korb DR, Greiner JV. Increase in tear film lipid layer thickness following treatment with warm compresses in patients with meibomian gland dysfunction. Eye Contact Lens. 2003;29(2):96-99. doi:10.1097/01.ICL.0000060998.20142.8D

25. Masmali AM, Al-Shehri A, Alanazi SA, Abusharaha A, Fagehi R, El-Hiti GA. Assessment of tear film quality among smokers using tear ferning patterns. $J$ Ophthalmol. 2016;2016:8154315. doi:10.1155/2016/8154315

26. Masmali AM, Maeni YA, El-Hiti GA, Murphy PJ, Almubrad T. Investigation of ocular tear ferning in controlled and uncontrolled diabetic subjects. Eye Contact Lens. 2018;44(Suppl 2):S70-S75. doi:10.1097/ICL.0000000000000419

27. Masmali AM, Alotaibi AG, Alanazi SA, Fagehi R, Abusharaha A, El-Hiti GA. The acute effect of a single dose of green tea on the quality and quantity of tears in normal eye subjects. Clin Ophthalmol. 2019;13:605-610. doi:10.2147/OPTH.S201127

28. Masmali AM, Alanazi SA, Almagren B, El-Hiti GA. Assessment of the tear film in normal eye subjects after consumption of a single dose of hot peppermint drink. Clin Optom. 2019;11:39-45. doi:10.2147/OPTO.S206904
29. Alanazi SA, El-Hiti GA, Al-Baloud AA, et al. Effects of short-term oral vitamin A supplementation on the ocular tear film in patients with dry eye. Clin Ophthalmol. 2019;13:599-604. doi:10.2147/ OPTH.S198349

30. Masmali AM, Al-Bahlal JM, El-Hiti GA, et al. Repeatability and diurnal variation of tear ferning test. Eye Contact Lens. 2015;41:262267. doi:10.1097/ICL.0000000000000116

31. World Medical Association Declaration of Helsinki: ethical principles for medical research involving human subjects. JAMA. 2013;310 (20):2191-2194. doi:10.1001/jama.2013.281053

32. Cohen JW. Statistical Power Analysis for the Behavioral Sciences. Hillsdale NJ: Lawrence Erlbaum Associates; 1988.

33. Pandit JC, Nagyová B, Bron AJ, Tiffany JM. Physical properties of stimulated and unstimulated tears. Exp Eye Res. 1999;68(2):247-253. doi:10.1006/exer.1998.0600

34. Tiffany JM, Pandit JC, Bron AJ. Soluble mucin and the physical properties of tears. In: Sullivan DA, Dartt DA, Meneray MA, editors. Lacrimal Gland, Tear Film, and Dry Eye Syndromes 2. Boston, MA: Springer; 1998:229-234.

35. Fullard RJ, Snyder C. Protein levels in nonstimulated and stimulated tears of normal human subjects. Invest Ophthalmol Vis Sci. 1990;31 (6):1119-1126

36. Sia RK, Ryan DS, Howard RS, et al. Non-stimulated tear sample collection using polyvinyl alcohol (PVA) foam and polyester wick. Int $J$ Ophthalmol Clin Res. 2016;3:048. doi:10.23937/2378-346X/1410048

37. Acosta MC, Peral A, Luna C, Pintor J, Belmonte C, Gallar J. Tear secretion induced by selective stimulation of corneal and conjunctival sensory nerve fibers. Invest Ophthalmol Vis Sci. 2004;45(7):23332336. doi:10.1167/iovs.03-1366

38. Yasui T, Karita K, Izumi H, Tamai M. Correlation between vasodilatation and secretion in the lacrimal gland elicited by stimulation of the cornea and facial nerve root of the cat. Invest Ophthalmol Vis Sci. 1997;38(12):2476-2482.

39. Friedman NJ, Butron K, Robledo N, Loudin J, Baba SN, Chayet A. A nonrandomized, open-label study to evaluate the effect of nasal stimulation on tear production in subjects with dry eye disease. Clin Ophthalmol. 2016;10:795-804. doi:10.2147/OPTH.S101716

40. Brinton M, Chung JL, Kossler A, et al. Electronic enhancement of tear secretion. J Neural Eng. 2016;13(1):016006. doi:10.1088/17412560/13/1/016006

41. Kossler AL, Wang J, Feuer W, Tse DT. Neurostimulation of the lacrimal nerve for enhanced tear production. Ophthal Plast Reconstr Surg. 2015;31 (2):145-151. doi:10.1097/IOP.0000000000000234

42. Brinton M, Kossler AL, Patel ZM, et al. Enhanced tearing by electrical stimulation of the anterior ethmoid nerve. Invest Ophthalmol Vis Sci. 2017;58(4):2341-2348. doi:10.1167/iovs.16-21362

43. Cerretani CF, Radke CJ. Tear dynamics in healthy and dry eyes. Curr Eye Res. 2014;39(6):580-595. doi:10.3109/02713683.2013.859274
Clinical Optometry

\section{Publish your work in this journal}

Clinical Optometry is an international, peer-reviewed, open access journal publishing original research, basic science, clinical and epidemiological studies, reviews and evaluations on clinical optometry. All aspects of patient care are addressed within the journal as well as the practice of optometry including economic and business analyses. Basic and clinical research papers are published that cover

Submit your manuscript here: https://www.dovepress.com/clinical-optometry-journal all aspects of optics, refraction and its application to the theory and practice of optometry. The manuscript management system is completely online and includes a very quick and fair peer-review system, which is all easy to use. Visit http://www.dovepress.com/ testimonials.php to read real quotes from published authors. 